
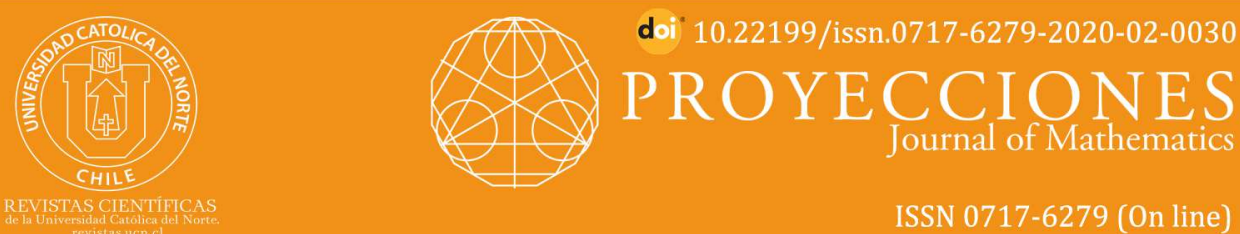

\title{
Fixed points and diametral sets for sequentially bounded mappings in orbital ultrametric spaces
}

M. Babahmed ${ }^{1}$ (1) orcid.org/0000-0003-1110-0982

A. El Amrani ${ }^{2}$

S. Lazaiz ${ }^{3}$ (D) orcid.org/0000-0001-7007-5233

${ }^{1}$ University Moulay Ismail, Dept. of Mathematics, Faculty of Sciences, Meknes, Morocco. m.babahmed@fs.umi.ac.ma University Sidi Mohamed Ben Abdellah, Dept. of Mathematics, FSDM, LAMA, Fes, Morocco.

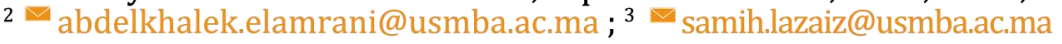

Received: June 2019 | Accepted: August 2019

\section{Abstract:}

In this paper, the $T$-orbital ultrametric spaces are introduced and a fixed point theorem for sequentially bounded mappings is given. Our main result extends some known theorems for nonexpansive mappings. Examples are given to support our work.

Keywords: Ultrametric spaces; T -orbital sets; T -dimetral sets; Fixed point; Sequentially bounded mappings.

MSC (2010): 47H10, 47H09; 54E40.

\section{Cite this article as (IEEE citation style):}

M. Babahmed, A. El Amrani, and S. Lazaiz, "Fixed points and diametral sets for sequentially bounded mappings in orbital ultrametric spaces", Proyecciones (Antofagasta, On line), vol. 39, no. 2, pp. 481-493, Apr. 2020, doi: 10.22199/issn.0717-6279-2020-02-0030.

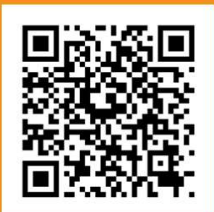

Article copyright: (C) 2020 Mohammed Babahmed, Abdelkhalek El Amrani and Samih Lazaiz. This is an open access article distributed under the terms of the Creative Commons Licence, which permits unrestricted use and distribution provided the original author and source are credited.

(cc) BY 


\section{Introduction}

Ultrametric spaces are a special type of metric spaces in which the triangle inequality is strengthened to a stronger triangular inequality. It happens that the theory of ultrametric spaces is closely related to a various directions of studies in mathematics, physics and computer sciences. This theory emerged in the last fifty years as a major field of studies in different branches of theoretical and applied mathematics $[4,5,12,13]$. For example it appears naturally in the study of pseudo-differential equations, more details are given in the recent book on ultrametric pseudo-differential equations and its applications [7].

The Banach contraction Principle [1], is the most fundamental result in fixed point theory. This result was extended and generalized in different ways, see $[2,3,9]$ and references therein for more details.

The study of fixed point theorems in ultrametric spaces find its root in the work of Petalas and Vidalis [11]. This theorem asserts that every nonexpansive mapping in spherically complete ultrametric space has a fixed point or a minimal $T$-invariant ball. Recently, Kirk and Shahzad in [8] have extended this result by noticing that any ball of the form $B(x, d(x, T x))$ contains either a fixed point or a minimal $T$-invariant ball. Note that the proof of Petalas and Vidalis is based on Zorn's lemma, while Kirk and Shahzad gave a constructive proof without invoking Zorn's lemma.

Priess-Crampe and Ribenboim in [14] recently established the Banach Contraction principle in generalized ultrametric spaces and were able to come up with applications in Logic programming (for more in the subject we refer the reader to $[4,13,12])$.

In this paper, we introduce a new class of mappings called sequentially bounded mappings which is properly larger than the class of nonexpansive mappings, and generalizes many other known classes of mappings. Supported by an example we establish an extension of Petalas and Vidalis theorem. Finally, we conclude by an open question about a fixed point theorem for sequentially bounded mappings in spherically complete ultrametric spaces which may leads to further investigations in the subject. 


\section{Preliminaries and basic results}

A metric space $(M, d)$ is said to be ultrametric if and only if the strong triangle inequality

$$
d(x, z) \leq \max \{d(x, y) ; d(y, z)\}
$$

holds for every $x, y, z \in M$. For each $x \in M$ and $r>0$, the closed ball in $M$ with center $x$ and radius $r$ is defined by $B(x, r)=\{y \in M ; d(x, y) \leq r\}$. Some fundamental properties satisfied by ultrametric spaces are that all triangles are isosceles, every ball is open and closed in the topological sense and the distance between points of disjoint balls is constant. For the latter. Let $B_{1}$ and $B_{2}$ be disjoint balls. Then, for $x \in B_{1}$ and $y \in B_{2}$, the number $d(x, y)$ is constant. Indeed, let $x \in B_{1}$ and $y, z \in B_{2}$; assume that $d(x, y) \neq d(x, z)$ by the isosceles triangle principle we get $x \in B_{2}$, which is in contradiction with the fact that $B_{1} \cap B_{2}=$.

Let $K$ be a bounded subset of $M$, we denote by $\delta(K)$ the diameter of $K$ that is

$$
\delta(K)=\sup \{d(x, y): x, y \in K\}
$$

Definition 2.1. Let $T: M \rightarrow M$ be a mapping and $K$ a bounded subset of $M$. We say that $K$ is $T$-diametral if:

- $T(K) \subset K$, i.e. $T$-invariant and;

- $d(y, T y)=\delta(K)$ for any $y \in K$.

Note that this definition mimic the notion of minimal $T$-invariant set in the classical case, except that in the non-archimedean case, the convex structure is never normal. The interested reader can consult the book of Khamsi and Kirk [6] for more on the subject.

Recall that for $x \in M$, the orbit of $x$ with respect to $T$ is the set

$$
\operatorname{Orb}(x, T)=\left\{T^{n} x: n \in \mathbf{N}\right\}
$$

Definition 2.2. $\quad$ (i) $A$ point $x$ in $M$ is said to be $T$-orbital if $O r b(x, T) \subset$ $B(x, d(x, T x))$.

(ii) A subset $K$ of $M$ is $T$-orbital, if $x$ is $T$-orbital for each $x \in K$. 
Remark 2.3. Note that if $T: K \rightarrow K$ is a nonexpansive mapping, that is

$$
d(T x, T y) \leq d(x, y) \quad \text { for any } x, y \in K,
$$

then the subset $K$ is $T$-orbital. Indeed, Let $x \in K$ and $n \in \mathbf{N}$, then

$$
\begin{aligned}
d\left(x, T^{n} x\right) & \leq \max \left\{d(x, T x), d\left(T x, T^{2} x\right), \ldots, d\left(T^{n-1} x, T^{n} x\right)\right\} \\
& \leq d(x, T x) .
\end{aligned}
$$

Thus $\operatorname{Orb}(x, T) \subset B(x, d(x, T x))$. This property is purely non-archimedean.

Definition 2.4. We will say that $M$ satisfies the $\mathcal{R C}$-property if any decreasing sequence $\left\{F_{n}\right\}_{n}$ of nonempty closed subsets of $M$, has a nonempty intersection in $M$.

Since a topological space is compact iff it has the finite intersection property, we have the following result.

Proposition 2.5. Each compact ultrametric space has the $\mathcal{R C}$-property.

Now, we define a new class of mappings which generalize many mapping classes.

Definition 2.6. A mapping $T: M \rightarrow M$ is said to be sequentially bounded if for each sequence $\left\{x_{n}\right\}_{n}$ which converges to $x$ with $x_{n} \neq x$ for all $n \in \mathbf{N}$, there exists $y \in M$ such that :

$$
T x_{n} \rightarrow y \quad \text { and } \quad d(x, T x) \leq d(x, y) .
$$

Remark 2.7. 1. Every continuous mapping is trivially sequentially bounded.

2. Every mapping with closed graph is sequentially bounded.

Next, we give an example of sequentially bounded mapping which is not continuous.

Example 2.8. Let $M=\mathbf{N}$ and define a distance $d$ on $M$ as follows:

$$
d(n, m)=\left\{\begin{array}{ll}
0 & \text { if } n=m \\
\frac{1}{m} & \text { if } m \geq 1 \text { and } n=0 \\
\frac{1}{n} & \text { if } n \geq 1 \text { and } m=0 \\
\max \left\{\frac{1}{n}, \frac{1}{m}\right\} & \text { otherwise. }
\end{array}\right\}
$$


$(M, d)$ is an ultrametric space. Let $T: M \rightarrow M$ defined by

$$
n \mapsto T n= \begin{cases}0 & \text { if } n=0 \\ 1 & \text { if } n \geq 1\end{cases}
$$

$T$ is not a continuous map. Indeed, let $\left\{x_{n}\right\}_{n} \subset \mathbf{N}^{*}$ such that $x_{n} \rightarrow 0$, and since $T x_{n}=1$ for all $n \in \mathbf{N}$ we have $T x_{n} \rightarrow 1 \neq T 0$.

$T$ is sequentially bounded mapping. Let $\left\{x_{n}\right\}_{n} \subset M$ and $x \in M$ such that $x_{n} \rightarrow x$ and $x_{n} \neq x$ for all $n$. We show that $\left\{T x_{n}\right\}_{n}$ converge to $y$ in $(M, d)$ and $d(x, T x) \leq d(x, y)$. It suffices to suppose that $x=0$, since if $x \neq 0$ the sequence $\left\{x_{n}\right\}_{n}$ will be eventually constant sequence. Let $x=0$. We have $T x_{n}=1$ for all $n$, then $T x_{n} \rightarrow 1$. Thus:

$$
d(x, T x)=d(0,0) \leq d(0,1)=d(x, y) .
$$

\section{Main result}

In the remaining part of this paper $M=(M, d)$ is an ultrametric space. The following example is due to Kirk and Shahzad [8]:

Example 3.1. Let $M=\{a, b, c, d\}$ with $d(a, b)=d(c, d)=\frac{1}{2} ; d(a, c)=$ $d(a, d)=d(b, c)=d(b, d)=1$. Then $(M, d)$ is an ultrametric space with the $\mathcal{R C}$-property. Define $T a=c ; T c=a ; T b=d ; T d=b$. Then $T$ is nonexpansive, $T$ does not have any fixed points.

This simple example shows that although $(M, d)$ is compact ultrametric space and $T$ is nonexpansive, there is no fixed point.

Before we go further we need some notations. Let $x$ be in $M, r \geq 0$ and $T: M \rightarrow M$ be a mapping. Set:

$$
\begin{aligned}
& C_{r}^{x}=\bigcap_{i \geq 0} B\left(T^{i} x, r\right) \\
& A_{r}^{x}=\left\{y \in C_{r}^{x}: d(y, T y) \leq r\right\} \\
& F_{x}=\left\{r \geq 0: A_{r}^{x} \neq\right\} .
\end{aligned}
$$

The following technical lemmas will be used in the proof of the main result (see Theorem 3.2 below).

Lemma 3.1. If $M$ is $T$-orbital, then $F_{x} \neq$. 
Proof. Let $x \in M$, set $r_{x}=d(x, T x)$. Since $\operatorname{Orb}(x, T) \subset B\left(x, r_{x}\right)$, we have

$$
x \in B\left(T^{i} x, r_{x}\right) \Rightarrow x \in C_{r_{x}}^{x}
$$

hence we have also $x \in A_{r_{x}}^{x}$ and $r_{x} \in F_{x}$, which implies that $F_{x} \neq$.

Lemma 3.2. Assume that $M$ is T-orbital. Then, for all $x \in M$, inf $F_{x} \in$ $F_{x}$.

Proof. Let $r_{1}=\inf F_{x}$, it is enough to show that $A_{r_{1}}^{x} \neq$. Since $r_{1}=$ $\inf F_{x}$, there exists a sequence $\left\{r_{n}\right\}$ of $F_{x}$ such that for each $n \geq 1$ we have :

$$
r_{1} \leq r_{n} \leq r_{1}+\frac{1}{n}
$$

Since $r_{n} \in F_{x}$ then $A_{r_{n}}^{x} \neq$. Let $z_{n} \in A_{r_{n}}^{x}$ for each $n \geq 1$, thus

$$
d\left(z_{n}, T z_{n}\right) \leq r_{n} \quad \text { and } \quad d\left(z_{n}, T^{i} x\right) \leq r_{n}(\forall i \in \mathbf{N})
$$

In particular we have

$$
d\left(z_{n}, x\right) \leq r_{n} \quad \text { and } \quad d\left(z_{n}, T x\right) \leq r_{n}
$$

thus $d\left(x, T^{x}\right) \leq r_{n}$ for all $n \geq 1$. So taking the limit with respect to $n$ yields

$$
d(x, T x) \leq r_{1} .
$$

On the other hand, for each $i, n \geq 1$

$$
\begin{aligned}
d\left(x, T^{i} x\right) & \leq \max \left\{d\left(x, y_{n}\right), d\left(y_{n}, T^{i} x\right)\right\} \\
& \leq r_{n}
\end{aligned}
$$

then $d\left(x, T^{i} x\right) \leq r_{1}$ for all $i \in \mathbf{N}$. Thus $x \in C_{r_{1}}^{x}$ and therefore $x \in A_{r_{1}}^{x}$. Hence $A_{r_{1}}^{x} \neq$ and $r_{1} \in F_{x}$.

Lemma 3.3. For each $x \in M$ and $r \geq 0, A_{r}^{x}$ is closed subset of $M$ provided that $T$ is sequentially bounded map.

Proof. Let $\left\{y_{n}\right\} \subset A_{r}^{x}$ such that $y_{n} \rightarrow y$, we may assume that $y_{n} \neq y$ for each $n \in \mathbf{N}$. Note that since $y_{n} \in C_{r}^{x}$ and $C_{r}^{x}$ is closed (as intersection of closed balls) we get $y \in C_{r}^{x}$. It suffices to show that $d(y, T y) \leq r$. Since $T$ is sequentially bounded we have

$$
T y_{n} \rightarrow z \quad \text { for some } z \in M \text {. }
$$


Then we get, $d(y, T y) \leq d(y, z)$. Since $\left\{y_{n}\right\}$ and $\left\{T y_{n}\right\}$ are convergent sequences, there exists $n_{0} \in \mathbf{N}$ such that for each $n \geq n_{0}$ we have

$$
d\left(y_{n}, y\right) \leq r \text { and } d\left(T y_{n}, z\right) \leq r
$$

thus for each $n \geq n_{0}$

$$
\begin{aligned}
d(y, z) & \leq \max \left\{d\left(y, y_{n}\right) ; d\left(y_{n}, T y_{n}\right) ; d\left(T y_{n}, z\right)\right\} \\
& \leq r
\end{aligned}
$$

and since $d(y, T y) \leq d(y, z)$, we get $y \in A_{r}^{x}$. Consequently $A_{r}^{x}$ is closed.

Now, we are able to state the main result of this paper.

Theorem 3.2. Let $(M, d)$ be an ultrametric space and let $T: M \rightarrow M$ be a sequentially bounded mapping. Then either $T$ has a fixed point or $M$ contains a $T$-diametral set provided that $M$ is $T$-orbital and satisfies the $\mathcal{R C}$-property.

Proof. Let $r_{1}$ be as in the proof of Lemma 3.2 and set $\alpha_{1}=\inf \{d(y, T y)$ : $\left.y \in A_{r_{1}}^{x}\right\}$. For each $y \in A_{r_{1}}^{x}$ we have

$$
\alpha_{1} \leq d(y, T y) \leq r_{1}
$$

Here we have two cases, either $\alpha_{1}=r_{1}$ or $\alpha_{1}<r_{1}$.

[1] Assume that $\alpha_{1}=r_{1}$, then we get

$$
d(y, T y)=r_{1} \quad \forall y \in A_{r_{1}}^{x} .
$$

[i] $A_{r_{1}}^{x}$ is $T$-invariant. Let $z \in A_{r_{1}}^{x}$, we have $z \in C_{r_{1}}^{x}$ and $d(z, T z) \leq r_{1}$. We prove that

$$
T z \in C_{r_{1}}^{x} \quad \text { and } \quad d\left(T z, T^{2} z\right) \leq r_{1} .
$$

Since $z \in C_{r_{1}}^{x}$, we get $d\left(z, T^{i} x\right) \leq r_{1}$ for each $i \in \mathbf{N}$ and hence we have

then $T z \in C_{r_{1}}^{x}$.

On the other hand,

$$
d\left(T z, T^{2} z\right) \leq \max \left\{d(T z, z), d\left(z, T^{2} z\right)\right\}
$$

and since $\operatorname{Orb}(z, T) \subset B(z, d(z, T z)$, we get

$$
d\left(z, T^{2} z\right) \leq d(z, T z) .
$$


So $d\left(T z, T^{2} z\right) \leq r_{1}$, thus $T z \in A_{r_{1}}^{x}$. have

[ii] $A_{r_{1}}^{x} \subset B\left(z, r_{1}\right)$ for each $z \in A_{r_{1}}^{x}$. Let $z \in A_{r_{1}}^{x}$. For all $y \in A_{r_{1}}^{x}$ we

$$
\begin{aligned}
d(y, z) & \leq \max \{d(y, x), d(x, z)\} \\
& \leq r_{1}
\end{aligned}
$$

since $y, z \in C_{r_{1}}^{x}$ that is $d(y, x) \leq r_{1}$ and $d(z, x) \leq r_{1}$. Thus

$$
A_{r_{1}}^{x} \subset B\left(z, r_{1}\right) \quad\left(\forall z \in A_{r_{1}}^{x}\right) .
$$

Consequently $\delta\left(A_{r_{1}}^{x}\right) \leq r_{1}$ and as $\alpha_{1}=r_{1}$ and $A_{r_{1}}^{x}$ is $T$-invariant, we obtain that

$$
\delta\left(A_{r_{1}}^{x}\right)=r_{1} .
$$

- If $r_{1}=0$, then $A_{r_{1}}^{x}=\{x\}$ and hence $T x=x$;

- If $r_{1}>0$. Then by equation (3.1), we have for all $y \in A_{r_{1}}^{x}$

$$
d(y, T y)=r_{1}=\delta\left(A_{r_{1}}^{x}\right)
$$

thus, $A_{r_{1}}^{x}$ is $T$-diametral.

[2] Now, assume that $r_{1}>0$ and $\alpha_{1}<r_{1}$. Since $\alpha_{1}$ is an infimum there exists $n_{2}>1$ and $y_{n_{2}} \in A_{r_{1}}^{x}$ such that

$$
\alpha_{1} \leq d\left(y_{n_{2}}, T y_{n_{2}}\right)<\alpha_{1}+\frac{1}{n_{2}} \leq r_{1}
$$

Set $r_{n_{2}}:=d\left(y_{n_{2}}, T y_{n_{2}}\right)$. Since $M$ is $T$-orbital, we have $y_{n_{2}} \in C_{r_{n_{2}}}^{y_{n_{2}}}$ and therefore $y_{n_{2}} \in A_{r_{n_{2}}}^{y_{n_{2}}}$ and $r_{n_{2}} \in F_{y_{n_{2}}}$. Let

$$
\begin{aligned}
r_{n_{2}}^{\prime} & =\inf F_{y_{n_{2}}} \\
\alpha_{n_{2}} & =\inf \left\{d(y, T y): y \in A_{r_{n_{2}}}^{y_{n_{2}}}\right\}
\end{aligned}
$$

thus we have, $\alpha_{n_{2}} \leq d(y, T y) \leq r_{n_{2}}$ for all $y \in A_{r_{n_{2}}}^{y_{n_{2}}}$.

As before, if $r_{n_{2}}=\alpha_{n_{2}}$ either $y_{n_{2}}$ is a fixed point of $T$ or $A_{r_{n_{2}}}^{y_{n_{2}}}$ is a $T$-diametral set in $M$.

In both cases, if this process terminates with a finite number of operations, the result follows. Otherwise, we assume that this process does not end, then there exists a strictly increasing sequence $\left\{n_{p}\right\}_{p}$ in $\mathbf{N}$ and a sequence $\left\{y_{n_{p}}\right\}$ in $M$ such that for all $p \in \mathbf{N}$, we have : 


$$
\begin{aligned}
F_{y_{n_{p}}} & \neq \\
r_{n_{p}}^{\prime} & =\inf F_{y_{n_{p}}} \\
\alpha_{n_{p}} & =\inf \left\{d(y, T y): y \in A_{r_{n_{p}}}^{y_{n_{p}}}\right\} \\
\alpha_{n_{p}} & <r_{n_{p}} \\
\alpha_{n_{p}} & \leq d(y, T y)<r_{n_{p}} \quad\left(\forall y \in A_{r_{n_{p}}}^{y_{n_{p}}}\right) \\
\alpha_{n_{p}} & <\alpha_{n_{p}}+\frac{1}{n_{p+1}} \leq r_{n_{p}} \\
r_{n_{p+1}} & \leq r_{n_{p}} .
\end{aligned}
$$

Now, since $r_{n_{p+1}} \leq r_{n_{p}}$ for each $p$ in $\mathbf{N}$, we have

$$
A_{r_{n_{p+1}}}^{y_{n_{p+1}}} \subset A_{r_{n_{p}}}^{y_{n_{p}}}
$$

and then, $\left\{A_{r_{n_{p}}}^{y_{n_{p}}}\right\}_{p}$ is a decreasing sequence of closed subsets of $M$ (Lemma 3.3). Using the $(\mathcal{R C})$-property we get

$$
A_{\infty}:=\bigcap_{p} A_{n_{p}} \neq
$$

Since $\left\{r_{n_{p}}\right\}_{p}$ is a decreasing sequence and $\left\{\alpha_{n_{p}}\right\}_{p}$ is an increasing and bounded above by $r_{1}, r=\lim _{p} r_{n_{p}}$ and $\alpha=\lim _{p} \alpha_{n_{p}}$ exist.

Let $z \in A_{\infty}$. Then for all $p \geq 1$

$$
\begin{aligned}
z \in A_{r_{n_{p}}}^{y_{n_{p}}} & \Rightarrow d(z, T z) \leq r_{n_{p}} \\
& \Rightarrow d(z, T z) \leq r .
\end{aligned}
$$

On the other hand, for all $p \geq 1$ we have

$$
\alpha_{n_{p}} \leq d(z, T z) \leq r \leq r_{n_{p}}<\alpha_{n_{p}}+\frac{1}{n_{p+1}}
$$

Taking the limit with respect to $p$ yields

$$
d(z, T z)=\alpha=r
$$

- If $r=0$, then $A_{\infty}=\{z\}$ and hence $T z=z$;

- If $r>0$. Since $A_{r_{n}}^{y_{n_{p}}}$ is $T$-invariant for each $p \geq 1$, then $A_{\infty}$ is $T$ invariant, and we have for all $z \in A_{\infty}$

$$
d(z, T z)=r .
$$


Moreover, for all $y, z \in A_{\infty}$ and $p \geq 1$ we have

$$
\begin{aligned}
d(y, z) & \leq \max \left\{d\left(y, y_{n_{p}}\right) ; d\left(z, y_{n_{p}}\right)\right\} \\
& \leq r_{n_{p}}
\end{aligned}
$$

thus $d(y, z) \leq r$. Hence $\delta\left(A_{\infty}\right)=r$. Therefore, $A_{\infty}$ is $T$-diametral in $M$.

Example 3.3. Let $p$ be a prime number and let $M=\mathbf{Z}_{p}$ be the ring of the $p$-adic integers endowed with the $p$-adic valuation $|\cdot|_{p}$ (see [15] for more details). The $p$-adic valuation $|\cdot|_{p}$ defines an ultrametric $d_{p}$ by

$$
d_{p}(x, y):=|x-y|_{p} \quad\left(x, y \in \mathbf{Z}_{p}\right) .
$$

It is known that $\left(M, d_{p}\right)$ is a compact ultrametric space (see [10, Theorem 1.2.8]). Thus, using Proposition 2.5, $(M, d)$ has the $\mathcal{R C}$-property.

Let $r>0$ and set $U=B(0, r) \subset \mathbf{Z}_{p}$ with $1 \notin U$. Since the space $\mathbf{Z}_{p}$ is totally disconnected, the characteristic function $T$ defined by:

$$
T x=\left\{0, \quad \text { if } x \in U 1, \quad \text { if } x \in \mathbf{Z}_{p} \backslash U .\right.
$$

is continuous. Furthermore, $\mathbf{Z}_{p}$ is T-orbital. Indeed,

- If $x \in U$ then $T x=0$, hence

$$
\operatorname{Orb}(x, T)=\{x, 0,0, \ldots\} \subset B\left(x,|x-T x|_{p}\right) .
$$

- If $x \in \mathbf{Z}_{p} \backslash U$ then $T x=1$ and $T^{n} x=1$ for any $n \geq 1$, hence

$$
\operatorname{Orb}(x, T)=\{x, 1,1, \ldots\} \subset B\left(x,|x-T x|_{p}\right) .
$$

All assumptions of Theorem 3.2 are satisfied, and $T$ have a fixed point, namely $T 0=0$.

It is worth mentioning that $T$ is not a nonexpansive map. Indeed, let $y \in \mathbf{Z}_{p} \backslash U$ such that $|y|_{p}<1$. Thus,

$$
|T 0-T y|_{p}=|T y|_{p}=1>|0-y|_{p} .
$$

The following result can be seen as an extension and strengthening of the results of Kirk-Shahzad [8] and Petalas-Vidalis [11].

Corollary 3.4. Under the hypotheses of Theorem 3.2, with $T$ is a nonexpansive mapping. $T$ has either a fixed point or $M$ has a $T$-diametral set. 


\section{Concluding remarks}

Recall that an ultrametric space $(M, d)$ is spherically complete, if every shrinking sequence of balls in $M$ has a nonempty intersection, i.e. for any sequence $\left\{B\left(x_{n}, r_{n}\right)\right\}_{n \in \mathbf{N}}$ of closed balls such that $r_{n+1}<r_{n}$ and $B\left(x_{n+1}, r_{n+1}\right) \subset B\left(x_{n}, r_{n}\right)$, for any $n \in \mathbf{N}$, we have $\bigcap_{n \in \mathbf{N}} B\left(x_{n}, r_{n}\right) \neq$.

Proposition 4.1. Let $(M, d)$ be an ultrametric space. If $M$ has the $\mathcal{R C}$ property, then $M$ is spherically complete.

Proof. Since the closed balls are closed sets, the result follows.

Example 4.2. Let $M=\mathbf{N}$ and consider the discrete ultrametric distance on $M$,

$$
d(n, m)= \begin{cases}0 & \text { if } n=m \\ 1 & \text { if } n \neq m\end{cases}
$$

It is known than a complete discrete ultrametric space is spherically complete, thus $(M, d)$ is a spherically complete ultrametric space. But $M$ does not satisfy the $\mathcal{R C}$-property. Indeed, set for each $n \in \mathbf{N}$

$$
A_{n}=[n, \infty) .
$$

In this context, every Cauchy sequence and furthermore every convergent sequence is eventually constant sequence. Thus $A_{n}$ is closed subset of M. Moreover, we have for all $n \in \mathbf{N}$

$$
A_{n+1} \subset A_{n}
$$

but we have trivially $\bigcap_{n \in \mathbf{N}} A_{n}=\emptyset$, then $M$ does not satisfy the $\mathcal{R C}$-property.

Therefore, the following question arise naturally:

Question 4.3. It is possible to get Theorem 3.2 by assuming the spherically completeness of $M$ instead of the $\mathcal{R C}$-property ?

We conclude the paper by the following remark which may give a possible outcome for future researches.

Remark 4.4. We have used the sequential boundedness of the mapping $T$ to prove that the sets $A_{r}^{x}$ are closed. So, in order to give a more generalized version, it is enough to find a class of mappings which generalizes the class of sequentially bounded ones, and which realizes the closedness of the sets $A_{r}^{x}$. 


\section{References}

[1] S. Banach, "Sur les opérations dans les ensembles abstraits et leur application aux équations intégrales", Fundamenta mathematicae, vol. 3, pp. 133-181, 1922, doi: 10.4064/fm-3-1-133-181.

[2] V. Berinde, Iterative approximation of fixed points, vol. 1912. Berlin: Springer, 2007, doi: 10.1007/978-3-540-72234-2.

[3] A. Granas and J. Dugundji, Fixed point theory. New York, NY: Springer, 2010,doi: 10.1007/978-0-387-21593-8.

[4] P. Hitzler and A. K. Seda, "The fixed-point theorems of priess-crampe and ribenboim in logic programming", in Valuation theory and its applications, vol. 1, F.- V. Kuhlmann, S. Kuhlmann, and M. Marshall, Eds. Providence, RI: American Mathematical Society, 2002, pp. 219-235, doi: 10.1090/fic/032.

[5] B. Hughes, "Trees and ultrametric spaces: a categorical equivalence", Advances in mathematics, vol. 189, no. 1, pp. 148-191, Dec. 2004, doi: 10.1016/j.aim.2003.11.008.

[6] M. A. Khamsi and W. A. Kirk, An Introduction to metric spaces and fixed point theory. New York, NY: John Wiley and sons, 2001, doi: 10.1002/9781118033074.

[7] A. Y. Khrennikov, S. V. Kozyrev, and W. A. Zúñiga-Galindo, Ultrametric pseudodifferential equations and applications, vol. 168. Cambridge: Cambridge University Press, 2018, doi: 10.1017/9781316986707.

[8] W. A. Kirk and N. Shahzad, "Some fixed point results in ultrametric spaces", Topology and its applications, vol. 159, no. 15, pp. 3327-3334, Sep. 2012, doi: 10.1016/j.topol.2012.07.016.

[9] W. A. Kirk and N. Shahzad, Fixed point theory in distance spaces. Cham: Springer, 2014, doi: 10.1007/978-3-319-10927-5.

[10] C. Perez-Garcia and W. H. Schikhof, Locally convex spaces over non- archimedean valued field, vol. 119. Cambridge: Cambridge University Press, 2010, doi: 10.1017/CB09780511729959.

[11] C. Petalas and T. Vidalis, "A fixed point theorem in non-archimedean vector spaces", Proceedings of the American Mathematical Society, vol. 118, no. 3, pp. 819-821, 1993, doi: 10.1090/S0002-9939-19931132421-2.

[12] S. Priess-Crampe and P. Ribenboim, "Logic programming and ultrametric spaces", Rendiconti di matematica e delle sue applicazioni, vol. 19, no. 2, pp. 155-176, 1999. [On line]. Available: https://bit.ly/2Yh0RC7 
[13] S. Priess-Crampe and P. Ribenboim, "Ultrametric spaces and logic programming", The journal of logic programming, vol. 42, no. 2, pp. 59-70, Feb. 2000, doi: 10.1016/s0743-1066(99)00002-3.

[14] S. Priess-Crampe and P. Ribenboim, "Ultrametric dynamics", Illinois journal of mathematics, vol. 55, no. 1, pp. 287-303, 2011. [On line]. Available: https://bit.ly/2VNH7Eu

[15] A. C. M. van. Rooij, Non-archimedean functional analysis. New York, NY: M. Dekker, 1978. 